Martínez Serrano, M.E., Pérez Herrero, M.H. y Burguera Condón, J.L. (2022). Orientación para el Desarrollo de la Carrera en Educación Secundaria: una Revisión Sistemática. Revista de Investigación Educativa,40(1), 107-126. DOI: http://dx.doi.org/10.6018/rie.431491

\title{
Orientación para el Desarrollo de la Carrera en Educación Secundaria: una Revisión Sistemática
}

\section{Career Development in Secondary Education: a Systematic Review}

\author{
María Eugenia Martínez Serrano, María del Henar Pérez Herrero y Joaquín Lorenzo Burguera \\ Condon' \\ Universidad de Oviedo
}

\begin{abstract}
Resumen
La Orientación para el Desarrollo de la Carrera (ODC) es uno de los ámbitos fundamentales de la intervención en orientación porque aporta a las personas, particularmente a los adolescentes, estrategias pertinentes para la toma de decisiones a partir del autoconocimiento de las propias competencias y del conocimiento del mundo académico y laboral. En la actual situación de cambio y transición hacia modelos de trabajo inciertos y cambiantes, con un predominio creciente de las tecnologías, es preciso orientar a las futuras generaciones tomando como punto de partida sus necesidades reales en el presente. Por eso, se precisa disponer de instrumentos de recogida de información que incluyan las variables pertinentes. De ahí que este trabajo se haya planteado desde una revisión sistemática en bases de datos específicas de educación y psicología, con objeto de identificar investigaciones significativas en ODC en adolescentes de educación secundaria, bachillerato y formación profesional. El procedimiento de búsqueda ha proporcionado 38 documentos que son analizados de manera exhaustiva. Los resultados muestran la diversidad de enfoques, instrumentos, y dimensiones de análisis. Concluimos destacando la necesidad de diseñar instrumentos que recojan información cuantitativa y cualitativa y tengan en cuenta variables de contexto, pero también variables que recojan información referida al autoconocimiento, el conocimiento del mundo laboral, las habilidades y recursos para la toma de decisiones, etc., sin olvidar aspectos como el género, la equidad y la inclusión de distintos colectivos y personas especialmente vulnerables.
\end{abstract}

1 Correspondencia: Joaquín Lorenzo Burguera Condón, burguera@uniovi.es, Facultad de Formación del Profesorado y Educación, C/ Aniceto Sela, 1, 33005 Oviedo, Asturias. 
Palabras clave: desarrollo de la carrera, revisión sistemática, enseñanza secundaria, toma de decisiones.

\begin{abstract}
Career Development (CDG) is an essential intervention area in guidance because it provides people, particularly adolescents, with relevant strategies for decision-making based on self-knowledge of their own competencies and knowledge of the academic and labor world. In the current situation of change and transition towards uncertain and changing work models, with an increasing predominance of technologies, it is necessary to guide future generations by taking as a starting point their real needs in the present. Therefore, it is required to have information collection instruments that include the relevant variables. Thus, in this paper a systematic review in specific databases of education and psychology has been carried out, in order to identify significant research on CDG in adolescents from secondary education, high school and vocational training. The searching procedure has provided 38 documents that are thoroughly analyzed. The results show the diversity of approaches, instruments, and dimensions of analysis. We conclude by highlighting the need to design instruments that collect quantitative and qualitative data and that consider not only context variables but also variables related to self-knowledge, labor market knowledge, skills for decision-making, gender, equity and the inclusion of different groups, especially vulnerable people.
\end{abstract}

Keywords: career development, literature review, secondary education, decision making.

\title{
Introducción
}

Los cambios políticos, económicos, tecnológicos y sociales en los que nos encontramos inmersos en las últimas décadas han transformado el mundo del trabajo, hasta tal punto que algunas profesiones han desaparecido, la mayoría han experimentado profundas modificaciones y, al tiempo, han aparecido otras nuevas. Parece esperable que esta tendencia continúe en los años venideros, sobre todo teniendo en cuenta lo que está suponiendo la crisis sanitaria mundial, cuyos efectos negativos a nivel económico, social y en todos los ámbitos de las personas, están empezando a ser evidentes, sobre todo en los sectores de población más vulnerables. En este escenario, los empleadores demandan a los trabajadores competencias relacionadas con una cualificación específica para cada puesto de trabajo, pero también competencias transversales que hagan posible una continua adaptación, máxima flexibilidad y total disposición para el cambio. La orientación, y de forma específica la orientación profesional, debe ser consecuente con esta realidad y abandonar su carácter finalista para adoptar un enfoque plenamente procesual, una acción orientadora que se prolongue a lo largo de toda la vida profesional de las personas (Martínez-Clares y Martínez-Juárez, 2011), que les ayude a afrontar la incertidumbre (Rodríguez Moreno, 2013). Esta concepción humanística de la orientación profesional entendida como proceso educativo de acompañamiento de la persona a lo largo de la vida, sobre todo en momentos de cambio y transición, ha determinado, entre otras razones, que nos refiramos a ella con la expresión Orientación para el Desarrollo de la Carrera (en adelante ODC). 
La ODC hace referencia a un proceso cuya finalidad es potenciar el desarrollo de la carrera de las personas dotándoles de estrategias, recursos y actuaciones que contribuyan de forma positiva a su realización personal, educativa, profesional y social en consonancia con el medio que les rodea (Álvarez González y Bisquerra, 2017).

Se trata de un proceso integral de proyección vital (Álvarez Justel, 2019) que incluye las orientaciones y ayudas necesarias para que las personas, a partir de un profundo conocimiento de sí mismas, elaboren, potencien y empleen sus capacidades para alcanzar el máximo desarrollo personal y profesional en un contexto social concreto (Ambiel et al., 2018).

Cuando la ODC se dirige a adolescentes, la finalidad es promover que aprendan a gestionar sus propias capacidades y competencias (Planas, 2012), ya sea para continuar su formación académica, o para incorporarse al mundo laboral, pero, sobre todo, para desarrollar un proyecto personal de vida y favorecer el aprendizaje para tomar decisiones de forma responsable, ajustada y autónoma (Santana, Alonso y Feliciano, 2018). Esto es, para construirse como personas y como ciudadanos (Taveira y Rodríguez Moreno, 2010). De ahí que abarque diferentes elementos como el reconocimiento de metas, aspiraciones, intereses, capacidades, habilidades y valores personales (Santana, Feliciano y SantanaLorenzo, 2012), además de información sobre la oferta formativa y del mercado laboral.

Son diversos los factores que pueden afectar a las decisiones del alumnado adolescente acerca de su futuro: el grupo de iguales, el centro educativo, el contexto social y económico, etc. (Santana, Alonso y Feliciano, 2018). Asimismo, la cultura familiar desempeña un rol primordial en la construcción del itinerario académico-profesional del adolescente (Torío, Hernández y Peña, 2007; Olaosebikan y Olusakin, 2014). La acción orientadora en el ámbito del desarrollo de la carrera ha de tener en consideración, por tanto, los factores mencionados, porque pueden influir en el diseño y definición del proyecto de vida de cada persona. Este último, debe tener presente los intereses y capacidades propias, con objeto de que la intervención orientadora facilite al alumnado las herramientas necesarias para introducirse y desenvolverse eficazmente en el mundo del trabajo (Santana, Alonso y Feliciano, 2018). Es en este contexto donde la comunidad educativa y los profesionales que en ella intervienen desempeñan una labor esencial para que cada estudiante, desde edades tempranas, diseñe un proyecto vital que le permita alcanzar un desarrollo integral como persona y le sea posible poner en acción sus fortalezas y capacidades (Hernández Franco, 2004), y desde la reflexión, pueda tomar decisiones ajustadas a sus actitudes, competencias y habilidades personales. Así, los programas de ODC implementados en los centros educativos han de caracterizarse por ser procesos continuos de acompañamiento y mediación que exigen al alumnado desempeñar un rol activo en la construcción y desarrollo de su proyecto de vida y profesional (Martínez-Clares, Pérez-Cusó y MartínezJuárez., 2014; Lomelí-Parga, López-Padilla y Valenzuela-González, 2016).

Los estudios sobre ODC en las etapas educativas preuniversitarias han puesto de manifiesto la necesidad de desarrollar actuaciones orientadoras eficaces que garanticen el óptimo tránsito del alumnado a la vida adulta (Figuera, Rodríguez y Llanes, 2015). Algunos estudios se han centrado en analizar constructos vinculados a la planificación y construcción del proyecto de vida, en los factores que le afectan o en las limitaciones y barreras que impiden su desarrollo (Santana, Feliciano y Santana-Lorenzo, 2012; Bounds, 2017; Paixão y Gamboa, 2017; Abubakar, 2018; Ambiel et al., 2018; Karacan- 
Ozdemir, 2019). Otros estudios (Álvarez González, Figuera y Torrado, 2011) revelan que, a pesar de su denominación y sus planteamientos iniciales, la ODC se activa de manera esporádica e impersonal, en determinadas circunstancias, períodos o momentos que exigen al alumnado tomar una decisión a corto plazo. Así entendida, la ODC pierde su carácter procesual, para devenir en despersonalizada y puntual, induciendo al alumnado a tomar decisiones rápidas, irreflexivas y, con frecuencia, erradas. Para evitar esta situación, conviene aplicar intervenciones que optimicen la acción orientadora que los jóvenes reciben en educación secundaria, en general, y en particular en aquellos aspectos que atañen a su futuro académico, profesional o personal (Álvarez González y Bisquerra, 2017). Estos planteamientos deben contribuir a potenciar los procesos de madurez, planificación y toma de decisiones de los estudiantes atendiendo a sus capacidades, intereses, recursos y circunstancias personales y sociales (Savickas, 2012).

Para ello, y con objeto de que la ODC sea eficaz, ésta debe personalizarse y adaptarse a cada estudiante y responder, desde un enfoque inclusivo, a la diversidad y heterogeneidad del alumnado que convive en las aulas. Teniendo en cuenta todo lo anterior, se precisa realizar una revisión de la literatura científica que nos permita, desde el estado actual de la cuestión, avanzar en el conocimiento del tema y construir los instrumentos adecuados para recoger información sobre las necesidades del alumnado en diferentes etapas educativas, y especialmente en la adolescencia, por ser un momento del desarrollo crucial en la toma de decisiones personales, académicas y profesionales.

\section{Método}

\section{Objetivo}

El objetivo del presente estudio es realizar una revisión sistemática para analizar estudios empíricos sobre ODC en educación secundaria, publicados entre los años 2000-2019, con objeto de identificar las dimensiones y variables fundamentales para el diseño de instrumentos de recogida de información sobre ese ámbito de intervención.

\section{Procedimiento}

El estudio ha consistido en una revisión sistemática de la literatura científica para identificar las dimensiones y variables, así como los instrumentos de recogida de información cuantitativa y cualitativa que se han utilizado en las investigaciones más relevantes en ODC en educación secundaria obligatoria, bachillerato y formación profesional.

El proceso ha adaptado las fases y criterios propios de las revisiones sistemáticas (Fink, 2019), que cumplen los requisitos del método científico sobre objetividad, sistematización y replicabilidad (Frías-Navarro y Monterde-i-Bort, 2018): a) Justificación y objetivos de la revisión b) Pregunta de investigación c) Estrategia de búsqueda (criterios de inclusión y exclusión) d) Extracción de datos de las publicaciones y organización de la información e) Análisis de la información e informe de resultados.

La revisión se justifica en la necesidad de conocer el estado de la cuestión de la ODC en educación secundaria como objeto de estudio para adaptar los instrumentos de recogida de información para la intervención orientadora desde el ámbito escolar 
a la realidad cambiante de la sociedad actual. La pregunta de investigación se centra en conocer el enfoque de los trabajos, los instrumentos utilizados, sujetos, diseño de investigación, dimensiones y variables de estudio y resultados.

La búsqueda se ha realizado en bases de datos que contienen documentos sobre investigaciones en Educación y Psicología: ERIC, WOS, Scopus, ScienDirect Elsevier, SciELO Google Académico, Dialnet y ProQuest. Se han utilizado como términos de búsqueda: "orientación vocacional", "orientación profesional", "desarrollo de la carrera", "adolescentes", "educación secundaria" y sus correspondencias en inglés, "vocational counseling", "career development", "career counseling", "adolescents", "secondary education". Los campos de búsqueda han sido: título (TI), resumen (AB) o palabras clave (KW) y las ecuaciones de búsqueda: [("orientación vocacional” AND "orientación profesional" AND “desarrollo de la carrera" AND "adolescentes" AND “educación secundaria”)].

Los artículos han sido seleccionados según los criterios de inclusión siguientes: estudios sobre ODC realizados siguiendo diseños cuantitativos, cualitativos o mixtos; publicados durante las dos primeras décadas del siglo XXI; escritos en lengua española, inglesa, francesa, portuguesa e italiana; y abarcando a muestras de alumnado de educación secundaria, bachillerato y formación profesional. Los criterios de exclusión han sido: trabajos en los que no constase información sobre metodología o no hubieran pasado un proceso de revisión por pares: trabajos teóricos, tesis doctorales, informes, conferencias, etc.; trabajos publicados con anterioridad al año 2000, en otros idiomas diferentes a los considerados en los criterios de inclusión y centrados en alumnado universitario o de educación infantil o primaria. Estos criterios aparecen detallados en la tabla 1.

Tabla 1

Criterios de inclusión y exclusión en la revisión sistemática

\begin{tabular}{|c|c|c|}
\hline \multicolumn{3}{|c|}{ Criterios de } \\
\hline Aspectos & Inclusión & Exclusión \\
\hline Diseño & $\begin{array}{c}\text { Estudios cuantitativos y cualita- } \\
\text { tivos sobre Orientación para el } \\
\text { Desarrollo de la Carrera y sus } \\
\text { dimensiones como objeto central } \\
\text { de estudio }\end{array}$ & $\begin{array}{l}\text { Artículos teóricos, tesis doctorales, es- } \\
\text { tudios de revisión y capítulos de libro, } \\
\text { publicaciones de carácter normativo, di- } \\
\text { vulgativo, informes breves, conferencias, } \\
\text { actas de congresos, cartas y ensayos. }\end{array}$ \\
\hline Temporalidad & Publicaciones entre 2000 y 2019 & Publicaciones anteriores al año 2000 \\
\hline Idioma & $\begin{array}{l}\text { Artículos en español, inglés, } \\
\text { francés, portugués e italiano }\end{array}$ & Otros idiomas \\
\hline Participantes & $\begin{array}{l}\text { Estudiantes de Educación Se- } \\
\text { cundaria Obligatoria, Bachillerato } \\
\text { y Formación Profesional o combi- } \\
\text { nación de ellas }\end{array}$ & $\begin{array}{l}\text { Estudiantes en educación universitaria, } \\
\text { infantil y primaria u otras }\end{array}$ \\
\hline
\end{tabular}

Con los criterios de inclusión y exclusión referidos se realizó una primera revisión centrada en los títulos, palabras-clave y resúmenes, que permitió descartar los documentos 
que incumplían los criterios de inclusión o cumplían los de exclusión. Posteriormente, se procedió a la lectura y análisis de los documentos incorporando la información en una base de datos incluyendo: objetivo, etapa de referencia, tamaño y composición de la muestra, diseño de investigación, instrumentos, dimensiones de estudio y análisis de datos.

\section{Resultados}

La figura 1 muestra el diagrama de flujo del proceso de revisión sistemática desarrollado. En la primera fase se obtuvieron 1100 referencias. En las fases siguientes se ha procedido a eliminar referencias duplicadas, aplicar los criterios de inclusión y exclusión y a leer y analizar los documentos. Este proceso ha dado como resultado un total de 38 documentos que son sometidos a análisis en el presente trabajo.

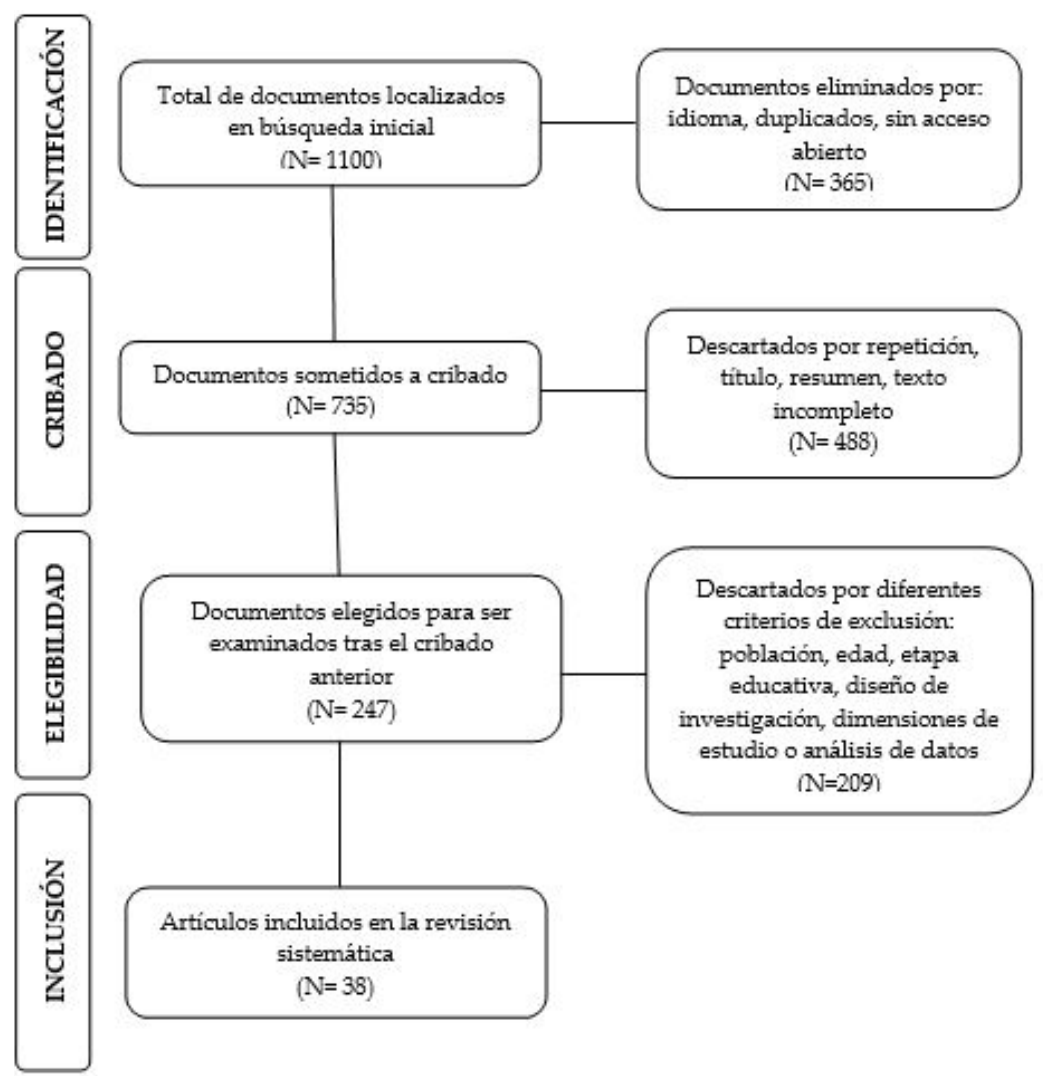

Figura 1. Diagrama de flujo de la revisión.

La tabla 2 muestra las claves numéricas utilizadas para identificar variables y contenidos de las referencias incluidas en la tabla 3, que presenta la información de los documentos recopilados en esta revisión. 
Tabla 2

Variables analizadas en la revisión sistemática. Claves numéricas

\begin{tabular}{cc}
\hline Variable & Código \\
\hline Objetivo & (1) Utilidad de los servicios de carrera; (2) Apoyos y barreras percibidas \\
en los planes de carrera; (3) Evaluación de necesidades de planificación de & carrera; (4) Autoeficacia de las decisiones de carrera y autoconcepto aca- \\
démico; (5) Decisión de la carrera respecto a la influencia de los padres y \\
niveles de apego parental; (6) Aspiraciones profesionales; (7) Impacto de la \\
condición de empleo/desempleo parental; (8) Necesidades de orientación \\
educativa, vocacional y asesoramiento; (9) Planes educativos y de carrera; \\
(10) Patrones de interacción familiar como predictores de la identidad \\
vocacional; (11) Toma de decisiones; (12) Exploración y planificación de la \\
carrera; (13) Adaptabilidad de la carrera; (14) Factores personales y ambi- \\
entales en el compromiso de elección de carrera; (15) Orientación Profe- \\
sional como guía en la elección vocacional; (16) Configuración de la carrera \\
profesional de alumnado de FP; (17) Participación en actividades de ori- \\
entación profesional; (18) Expectativas formativo-laborales; (19) Influencia \\
del capital cultural y familiar en la orientación académico-profesional; (20) \\
Factores contextuales que pueden ayudar u obstaculizar el desarrollo de la \\
carrera; (21) Gestión de las transiciones de carrera y autoconstrucción; (22) \\
Claridad del Proyecto de Vida; (23) Perfiles motivacionales
\end{tabular}

Etapa educativa

(1) Educación Secundaria; (2) Bachillerato; (3) Formación Profesional

Muestra

(1) Mixto (mujeres y hombres); (2) Mujeres; (3) Hombres; (4) Caso único;

(5) Estudiantes; (6) Docentes; (7) Padres

Diseño de investigación

(1) No experimental; (2) Experimental; (3) Cuasiexperimental; (4) Transversal; (5) Longitudinal; (6) De evolución de grupo cohorte; (7) Comparación de medidas pre y post test; (8) Descriptivo/Exploratorio

Instrumento/s

(1) Cuestionario; (2) Entrevista; (3) Grupos de discusión; (4) Otros documentos (notas, informes...)

Dimensiones de estudio

(1) Datos sociodemográficos; (2) Exploración y planificación de carrera; (3) Autoeficacia profesional; (4) Autoconcepto académico-profesional; (5) Conflictos internos/externos; (6) Madurez vocacional; (7) Elección de carrera; (8) Autonomía;(9) Influencia familiar; (10) Autoconocimiento; (11)

Aspiraciones de carrera; (12) Procesos de decisión vocacional; (13) Factores de estrés en el proceso de guía; (14) Autoconstrucción; (15) Transición de la carrera; (16) Expectativas formativo-laborales; (17) Orientación Profesional; (18) Expectativas académicas; (19) Opciones de carrera preferidas; (20) Compromiso con la carrera; (21) Claridad del Proyecto de Vida; (22) Motivación; (23) Habilidades, destrezas y capacidades; (24) Exigencias del mercado de trabajo; (25) Apoyos/barreras académicas, profesionales, vocacionales y familiares 


\begin{tabular}{cc}
\hline Variable & Código \\
\hline $\begin{array}{c}\text { Análisis de } \\
\text { datos }\end{array}$ & $\begin{array}{c}\text { (1) Análisis estadístico descriptivo; (2) Univariado/Bivariado; (3) Multivari- } \\
\text { ado; (4) Análisis de contenido; (5) Factorial confirmatorio; (6) Correlacio- } \\
\text { nal/Inferencial; (7) Discriminante }\end{array}$ \\
\hline
\end{tabular}

Los resultados obtenidos en la revisión que se exponen en la tabla 3 se comentan a continuación siguiendo los elementos analizados. Como puede constatarse, de los 38 estudios seleccionados en esta revisión, cumpliendo los criterios establecidos, en relación con el idioma, la mayoría son investigaciones realizadas en países europeos (19/38) y publicados en español (12/19), inglés (4/19) y portugués (3/19). También hay estudios realizados en países americanos (13/38), publicados en inglés $(7 / 13)$, portugués (1/13) y español (1/13); y trabajos desarrollados en países del continente africano (6/38) escritos en lengua inglesa (6/6).

Atendiendo al objetivo de la investigación, la mayoría (11/38) se centra en el proceso de toma de decisiones vocacionales (Moura y Silveira, 2002; Gibbons et al., 2006; Salami y Oyesoji-Aremu, 2007; Königstedt y Taveira, 2010; Hirschi, Niles y Akos, 2011; Santana y Feliciano, 2011; Marcionetti, 2014; Ventura y Noronha, 2014; Bounds, 2017; Ambiel et al., 2018; Karacan-Ozdemir, 2019). Otros estudios (8/38) se centran en identificar los apoyos, barreras y necesidades experimentadas por los jóvenes en el proceso de desarrollo de la carrera y su participación en actividades de orientación profesional (Witko et al., 2006; Lozano y Repetto, 2007; Bloxom et al., 2008; Dogar et al, 2011; Safta, 2015; Draaisma, Meijers y Kuijpers, 2017; Abubakar, 2018; Vázquez y Blanco, 2019). En algunos casos (7/38) analizan los factores de influencia contextuales y sociofamiliares que pueden incidir en el proceso de construcción del proyecto de vida de los estudiantes (Hargrove, Inman y Crane, 2005; Torío, Hernández y Peña, 2007; Cenkseven-Önder, Kirdok y Isik, 2010; Faria, 2013; Ferrari et al., 2015; Olaosebikan y Olusakin, 2014; Rodríguez-Menéndez, Peña-Calvo e Inda-Caro, 2016). También se han identificado estudios (6/38) que ponen el foco en el análisis de las diferencias en los objetivos académicos y profesionales y en las expectativas formativas y laborales del alumnado en función del sexo (Torío, Hernández y Peña, 2007; Tang, Pan y Newmeyer, 2008; Nuñez, 2010; Santana, Feliciano y Santana-Lorenzo, 2012; Bacanli, 2016; Dudovitz et al., 2017). Asimismo, se han recuperado estudios (5/38) que analizan la utilización y valoración de los servicios de orientación, así como la utilidad de determinadas metodologías en el proceso de autoconstrucción del proyecto de vida (Rodríguez-Menéndez, Torío-López y Fernández-García, 2006; Morán, Muñoz y García, 2015; Paixão y Gamboa, 2017; Rochat y Rossier, 2016; Maree, Cook y Fletcher, 2018).

En relación con la etapa educativa son mayoría los que se centran en educación secundaria y bachillerato y muy escasos en formación profesional. La totalidad de los estudios que cumplen los requisitos establecidos, treinta y ocho, incluyen estudiantes de Educación Secundaria Obligatoria (ESO), Bachillerato y Formación Profesional. Así pues, catorce de los 38 estudios centran la investigación de forma exclusiva en alumnado escolarizado en Educación Secundaria o su equivalente (Rodríguez-Menéndez, Torío-López y Fernández-García, 2006; Torío, Hernández y Peña, 2007; Tang, Pan y 
Newmeyer, 2008; Ferrari et al., 2015; Königstedt y Taveira, 2010; Cenkseven-Önder, Kirdok y Isik, 2010; Marcionetti, 2014; Morán, Muñoz y García, 2015; Dogar et al., 2011; Hirschi, Niles y Akos, 2011; Faria, 2013; Olaosebikan y Olusakin, 2014; Rochat y Rossier, 2016; Dudovitz et al., 2017). Otros estudios (diez) abarcan todas las enseñanzas básicas y medias, la Formación Profesional y el Bachillerato o equivalentes en cada país (Hargrove, Inman y Crane, 2005; Lozano y Repetto., 2007; Salami y OyesojiAremu, 2007; Santana, Feliciano y Santana-Lorenzo, 2012). En once trabajos los sujetos son estudiantes de secundaria y Bachillerato (Hargrove, Inman y Crane, 2005; Witko et al., 2006) y en cuatro, únicamente, de Bachillerato (Bloxom et al., 2008; Santana y Feliciano, 2011; Safta, 2015, Rodríguez-Menéndez, Peña-Calvo e Inda-Caro, 2016). Por último, tan sólo un estudio dedica su atención a los estudiantes de Formación Profesional (Nuñez, 2010).

En relación con la composición de la muestra, en todos los estudios se recoge información del alumnado, varones y mujeres; uno de ellos incluye, además, a los docentes (Olivares, de León y Gutiérrez, 2010) y otro añade a padres y madres (Rodríguez Menéndez, Torío López y Fernández García, 2006 como agente informador.

Teniendo en consideración el diseño de investigación de los estudios se refleja una homogeneidad de investigaciones de carácter no experimental (36/38), de los que 32 son de carácter transversal, dos longitudinales, y uno de tipo comparativo. Mayoritariamente (34/38), son estudios descriptivos. Por otro lado, tres investigaciones emplean un diseño cuasi-experimental, dos de ellas de corte longitudinal (Moura y Silveira, 2002; Königstedt y Taveira, 2010) y una de carácter comparativo-descriptivo (Maree, Cook y Fletcher, 2018).

Con respecto a los instrumentos empleados casi la totalidad de las investigaciones (37 estudios), han tomado como instrumento para recabar y apoyar sus datos el cuestionario o la encuesta.

Por último, en cuanto a las dimensiones de estudio, se han identificado en los estudios analizados, 25 dimensiones (tabla 2). La casi totalidad de los estudios explora varias dimensiones, si bien la dimensión más estudiada en la mayoría de ellos es la exploración y planificación de la carrera (16/38), seguida de aquella relativa a los procesos de decisión vocacional (9/38), del autoconcepto académico-profesional (8/38) y de los apoyos/barreras académicas, profesionales, vocacionales y familiares (7/38). También se han localizado en varios trabajos como dimensiones de estudio, la autoeficacia profesional (6/38), la influencia familiar (6/38), las aspiraciones de carrera (6/38), la orientación profesional (6/38), y las opciones de carrera preferidas (6/38). Además, otros estudios consideran los conflictos internos-externos (4/38), autoconstrucción (4/38), expectativas formativo-laborales (4/38), expectativas académicas (4/38), autoconocimiento $(3 / 3)$, compromiso con la carrera (3/38), claridad del proyecto de vida (3/38) y habilidades, destrezas y capacidades (3/38).

Son escasos los trabajos que incluyen en el estudio las exigencias del mercado laboral (Ferrari et al., 2015, y Núñez Barriopedro, 2010), la elección de carrera (Cenkseven-Önder, Kirdok y Isik, 2010; y Salami y Oyesoji-Aremu, 2007), la transición de la carrera (Karacan-Ozdemir, 2019); Maree, Cook, y Fletcher, L., 2018), la madurez vocacional (Inda-Caro, Rodríguez-Menéndez, y Torío-López, 2017; Santana Vega, Feliciano García y Santana Lorenzo, 2012), la autonomía (Cenkseven-Önder, 
Kirdok y Isik, 2010), los factores de estrés en el proceso de guía (Bacanli, 2016) y la motivación (Lozano y Repetto, 2007).

La escasa presencia de algunas de estas últimas dimensiones en las investigaciones sobre ODC, nos indican el camino a seguir en futuras investigaciones, entre otras razones, por la influencia que tienen en la construcción y desarrollo del proyecto de vida personal y profesional de los estudiantes, sobre todo en la etapa de la adolescencia, agentes como la familia (Torío López, Hernández García y Peña Calvo, 2007), y por los riesgos que conlleva una injerencia excesiva en las decisiones que estos estudiantes tienen que tomar de forma autónoma en función de sus propios intereses y preferencias, sin verse obligados por la necesidad de buscar la aprobación de otras personas (Torío López, Hernández García y Peña Calvo, 2007; Nuñez Barriopedro, 2010; Faria, 2013; Olaosebikan y Olusakin, 2014; Bacanli, 2016).

También será preciso tener presente que cuando se han investigado las dificultades, barreras y necesidades que presenta la ODC, se han identificado algunos aspectos que precisan mejorarse en el proceso orientador. Los estudios coinciden en señalar, desde el punto de vista de los propios alumnos, que las acciones orientadoras desarrolladas por los centros educativos resultan a menudo insuficientes provocando que los estudiantes desconozcan las posibilidades educativas y formativas a su alcance, lo cual provoca en ellos una acusada sensación de inseguridad e inestabilidad (Blossom et al., 2008; Dogar et al., 2011).

Cuando los estudios han incluido la perspectiva de género al analizar constructos como las expectativas formativas y laborales de los estudiantes, o los objetivos y claridad al configurar su Proyecto de Vida, los resultados obtenidos no han ido siempre en la misma dirección. Así, en alguno de ellos no se han registrado diferencias estadísticamente significativas entre ambos géneros (Santana Vega, Feliciano García y Santana Lorenzo, 2012), mientras que en otros estudios si se han registrado diferencias significativas en cuanto a las opciones académicas elegidas entre ambos géneros (Rodríguez Menéndez, Torío López y Fernández García, 2006; Tang, Pan y Newmeyer, 2008; Santana Vega, Feliciano García y Santana Lorenzo, 2012).

En relación con el análisis de los datos, la mayoría de los estudios dan cuenta de los análisis descriptivos realizados (36/38), univariados o bivariados (13/38) y multivariados (17/38). Algunos estudios (4/38) han combinado una metodología mixta cuantitativa (análisis estadístico) y cualitativa (análisis de contenido), utilizando cuestionarios, entrevistas, informes, etc. (Olivares, de León y Gutiérrez, 2010; Rodríguez Menéndez, Peña Calvo e Inda Caro, 2016; Rochat y Rossier, 2016; Dudovitz et al., 2017). Solo en una investigación sus autores han empleado una metodología cualitativa mediante la aplicación de una entrevista personal a la muestra objeto de estudio (Draaisma, Meijers y Kuijpers, 2018).

Como puede apreciarse, algunos trabajos examinados en esta revisión analizan diferentes constructos en un mismo estudio por lo que aparecen agrupados en varias de las categorías establecidas. 
Tabla 3

Referencias de orientación para el desarrollo de la carrera analizadas en la revisión sistemática

\begin{tabular}{|c|c|c|c|c|c|c|c|}
\hline Autores & Objetivo & Etapa & Muestra & Diseño & Instrumento & $\begin{array}{l}\text { Dimensión } \\
\text { de estudio }\end{array}$ & $\begin{array}{l}\text { Análisis } \\
\text { de datos }\end{array}$ \\
\hline $\begin{array}{c}\text { Abubakar, } \\
\text { (2018) }\end{array}$ & 17 & $1 ; 2$ & $1 ; 5$ & $1 ; 4 ; 8$ & 1 & $2 ; 17$ & $1 ; 5$ \\
\hline $\begin{array}{c}\text { Ambiel, } \\
\text { Moreira, } \\
\text { Oliveira, } \\
\text { Pereira y } \\
\text { Hernández, } \\
\text { (2018) }\end{array}$ & 4 & $1 ; 2$ & $1 ; 5$ & $1 ; 4 ; 8$ & 1 & $2 ; 3 ; 10 ; 11$ & $1 ; 3$ \\
\hline $\begin{array}{l}\text { Bacanli } \\
(2016)\end{array}$ & $3 ; 18 ; 22 ; 23$ & 1 & 1 & $1 ; 4 ; 5$ & 1 & $5 ; 13 ; 21$ & $1 ; 3 ; 6$ \\
\hline $\begin{array}{c}\text { Bloxom, } \\
\text { Bernes, } \\
\text { Magnus- } \\
\text { son, Gunn, } \\
\text { Bardick, Orr } \\
\text { y McKnight } \\
\text { (2008) }\end{array}$ & $1 ; 2 ; 3$ & $1 ; 2$ & $1 ; 5$ & $1 ; 4 ; 8$ & 1 & $1 ; 2$ & $1 ; 2$ \\
\hline $\begin{array}{c}\text { Bounds } \\
\text { (2017) }\end{array}$ & 4 & $1 ; 2$ & $1 ; 5$ & $1 ; 4 ; 8$ & 1 & $1 ; 3 ; 4$ & $1 ; 3$ \\
\hline $\begin{array}{l}\text { Cenkseven- } \\
\text { Önder, } \\
\text { Kırdök y } \\
\text { Işı1k (2010) }\end{array}$ & 5 & 2 & $1 ; 5$ & $1 ; 4 ; 8$ & 1 & $5 ; 7 ; 8 ; 9$ & $1 ; 2$ \\
\hline $\begin{array}{c}\text { Dogar, } \\
\text { Azeem, } \\
\text { Majoka, } \\
\text { Mehmood y } \\
\text { Latif (2011) }\end{array}$ & $2 ; 8$ & 2 & $1 ; 5$ & $1 ; 4 ; 8$ & 1 & 25 & $1 ; 2$ \\
\hline $\begin{array}{c}\text { Draaisma, } \\
\text { Meijers y } \\
\text { Kuijpers } \\
(2018)\end{array}$ & 17 & 3 & $1 ; 6$ & $1 ; 4 ; 8$ & 2 & 17 & 4 \\
\hline $\begin{array}{c}\text { Dudovitz, } \\
\text { Chung, Nel- } \\
\text { son y Wong } \\
\text { (2017) }\end{array}$ & 6 & 1 & $1 ; 5$ & $1 ; 4 ; 8$ & $1 ; 2 ; 3$ & $3 ; 11$ & $1 ; 3 ; 4$ \\
\hline Faria (2013) & 7 & 2 & $1 ; 5$ & $1 ; 4 ; 8$ & 1 & 12 & $1 ; 5$ \\
\hline
\end{tabular}




\begin{tabular}{|c|c|c|c|c|c|c|c|}
\hline Autores & Objetivo & Etapa & Muestra & Diseño & Instrumento & $\begin{array}{l}\text { Dimensión } \\
\text { de estudio }\end{array}$ & $\begin{array}{l}\text { Análisis } \\
\text { de datos }\end{array}$ \\
\hline $\begin{array}{c}\text { Ferrari, } \\
\text { Ginevra, } \\
\text { Santilli, } \\
\text { Nota, Sga- } \\
\text { ramella, } \\
\text { y Soresi } \\
(2015)\end{array}$ & $20 ; 21$ & 1 & $1 ; 5$ & $1 ; 5 ; 7 ; 8$ & 1 & $2 ; 14 ; 24$ & $1 ; 3$ \\
\hline $\begin{array}{c}\text { Gibbons, } \\
\text { Borders, } \\
\text { Wiles, } \\
\text { Stephan y } \\
\text { Davis (2006) }\end{array}$ & $9 ; 10 ; 11$ & $1 ; 2$ & $1 ; 5$ & $1 ; 4 ; 8$ & 1 & $1 ; 2$ & $1 ; 2 ; 4$ \\
\hline $\begin{array}{l}\text { Hargrove, } \\
\text { Inman } \\
\text { y Crane } \\
(2005)\end{array}$ & 13 & 1,2 & $1 ; 5$ & $1 ; 4 ; 8$ & 1 & $1 ; 2 ; 9$ & $1 ; 3$ \\
\hline $\begin{array}{c}\text { Hirschi, } \\
\text { Niles y } \\
\text { Akos (2011) }\end{array}$ & 14 & 2 & $1 ; 5$ & $1 ; 5 ; 6 ; 8$ & 1 & $2 ; 19 ; 20$ & $1 ; 3$ \\
\hline $\begin{array}{l}\text { Inda, Rodrí- } \\
\text { guez y Torío } \\
\quad(2017)\end{array}$ & $2 ; 20$ & 2 & $1 ; 5$ & $1 ; 4 ; 8$ & 1 & $1 ; 6 ; 10 ; 25$ & $1 ; 2 ; 5 ; 6$ \\
\hline $\begin{array}{l}\text { Karacan- } \\
\text { Ozdemir } \\
(2019)\end{array}$ & $11 ; 13$ & $1 ; 2$ & $1 ; 5$ & $1 ; 4 ; 8$ & 1 & $2 ; 12 ; 14 ; 15$ & $1 ; 5$ \\
\hline $\begin{array}{l}\text { Königstedt } \\
\text { y Taveira } \\
(2010)\end{array}$ & $11 ; 12$ & 2 & $1 ; 5$ & $3 ; 5 ; 7$ & 1 & $2 ; 5 ; 19$ & $1 ; 5 ; 6$ \\
\hline $\begin{array}{c}\text { Lozano y } \\
\text { Repetto } \\
(2007)\end{array}$ & $1 ; 2$ & $1 ; 2 ; 3$ & $1 ; 5$ & $1 ; 4 ; 8$ & 1 & $\begin{array}{c}4 ; 5 ; 12 ; 19 \\
22\end{array}$ & $1 ; 3$ \\
\hline $\begin{array}{l}\text { Marcionetti } \\
\text { (2014) }\end{array}$ & $11 ; 14$ & 1 & $1 ; 5$ & $1 ; 7 ; 8$ & 1 & $10 ; 12 ; 25$ & $1 ; 3 ; 6$ \\
\hline $\begin{array}{c}\text { Maree, } \\
\text { Cook y } \\
\text { Fletcher } \\
(2018)\end{array}$ & 21 & 2 & $1 ; 5$ & $3 ; 7 ; 8$ & 1 & $14 ; 15$ & $1 ; 5 ; 4$ \\
\hline $\begin{array}{l}\text { Morán, Mu- } \\
\text { ñoz y Gar- } \\
\text { cía (2015) }\end{array}$ & $1 ; 15$ & 2 & $1 ; 5$ & $1 ; 4$ & 1 & $16 ; 20 ; 23$ & $1 ; 3 ; 6$ \\
\hline $\begin{array}{c}\text { Moura y } \\
\text { Silveira } \\
(2002)\end{array}$ & 16 & 2 & $1 ; 5$ & $3 ; 5 ; 7$ & 1 & $17 ; 23$ & $1 ; 2 ; 5$ \\
\hline
\end{tabular}




\begin{tabular}{|c|c|c|c|c|c|c|c|}
\hline Autores & Objetivo & Etapa & Muestra & Diseño & Instrumento & $\begin{array}{l}\text { Dimensión } \\
\text { de estudio }\end{array}$ & $\begin{array}{l}\text { Análisis } \\
\text { de datos }\end{array}$ \\
\hline $\begin{array}{l}\text { Núñez } \\
\text { (2010) }\end{array}$ & 17 & 3 & $1 ; 5$ & $1 ; 4 ; 8$ & 1 & $2 ; 17 ; 23 ; 24$ & $1 ; 3 ; 5$ \\
\hline $\begin{array}{l}\text { Olaosebikan } \\
\text { y Olusakin } \\
\text { (2014) }\end{array}$ & 5 & 2 & $1 ; 5$ & $1 ; 4 ; 8$ & 1 & $12 ; 18$ & $1 ; 2$ \\
\hline $\begin{array}{c}\text { Olivares, } \\
\text { de León y } \\
\text { Gutiérrez } \\
(2010)\end{array}$ & $1 ; 8$ & 1 & $\begin{array}{c}1 ; \\
5 ; 6\end{array}$ & $1 ; 4 ; 8$ & $1 ; 2$ & $9 ; 19$ & $1 ; 2 ; 4$ \\
\hline $\begin{array}{c}\text { Paixão y } \\
\text { Gamboa } \\
(2017)\end{array}$ & $6 ; 23$ & $1 ; 2$ & $1 ; 5$ & $1 ; 4 ; 8$ & 1 & $2 ; 21$ & $1 ; 23$ \\
\hline $\begin{array}{c}\text { Rochat y } \\
\text { Rossier } \\
(2016)\end{array}$ & 18 & 2 & $1 ; 5$ & $1 ; 4 ; 8$ & $1 ; 2$ & $2 ; 4 ; 14 ; 19$ & $1 ; 2 ; 4 ; 8$ \\
\hline $\begin{array}{c}\text { Rodríguez- } \\
\text { Menéndez, } \\
\text { Torío-López } \\
\text { y Fernán- } \\
\text { dez- García } \\
\text { (2006) }\end{array}$ & 18 & 2 & $\begin{array}{l}1 ; 5 \\
6 ; 7\end{array}$ & $1 ; 4 ; 8$ & 1 & $\begin{array}{c}1 ; 4 ; 12 ; 16 \\
18\end{array}$ & $1 ; 5$ \\
\hline $\begin{array}{c}\text { Rodríguez- } \\
\text { Menéndez, } \\
\text { Peña-Calvo } \\
\text { y Inda-Caro } \\
\quad(2016)\end{array}$ & 20 & 1 & $1 ; 5$ & $1 ; 4 ; 8$ & $1 ; 4$ & $12 ; 25$ & 4 \\
\hline Safta, (2015) & 2 & 1 & $1 ; 5$ & $1 ; 4 ; 8$ & 1 & $\begin{array}{c}1 ; 2 ; 4 ; 9 \\
11 ; 17\end{array}$ & $1 ; 2 ; 4$ \\
\hline $\begin{array}{l}\text { Salami y } \\
\text { Oyesoji Are- } \\
\text { mu (2007) }\end{array}$ & 5 & 2 & $1 ; 5$ & $1 ; 4 ; 8$ & 1 & $\begin{array}{l}2 ; 7 ; 9 ; 11 \\
\quad 17 ; 20\end{array}$ & $1 ; 3$ \\
\hline $\begin{array}{c}\text { Santana y } \\
\text { Feliciano } \\
(2011)\end{array}$ & 4 & 1 & $1 ; 5$ & $1 ; 4 ; 8$ & 1 & $1 ; 12 ; 18$ & $1 ; 3$ \\
\hline $\begin{array}{l}\text { Santana, } \\
\text { Feliciano } \\
\text { y Santana- } \\
\text { Lorenzo } \\
\text { (2012) }\end{array}$ & $18 ; 22$ & $1 ; 2 ; 3$ & $1 ; 5$ & $1 ; 4 ; 8$ & 1 & $1 ; 3 ; 6 ; 11 ; 21$ & $1 ; 3$ \\
\hline $\begin{array}{c}\text { Tang, Pan y } \\
\text { Newmeyer } \\
(2008)\end{array}$ & $2 ; 3 ; 9 ; 12$ & 2 & $1 ; 5$ & $1 ; 4 ; 8$ & 1 & $3 ; 11 ; 18 ; 19$ & $1 ; 3 ; 6$ \\
\hline
\end{tabular}




\begin{tabular}{|c|c|c|c|c|c|c|c|}
\hline Autores & Objetivo & Etapa & Muestra & Diseño & Instrumento & $\begin{array}{l}\text { Dimensión } \\
\text { de estudio }\end{array}$ & $\begin{array}{l}\text { Análisis } \\
\text { de datos }\end{array}$ \\
\hline $\begin{array}{c}\text { Torío; } \\
\text { Hernández } \\
\text { y Peña } \\
\text { (2007) }\end{array}$ & 19 & 2 & $1 ; 5$ & $1 ; 4 ; 8$ & 1 & $2 ; 4 ; 16 ; 25$ & $1 ; 2$ \\
\hline $\begin{array}{c}\text { Vázquez } \\
\text { y Blanco } \\
(2019)\end{array}$ & $4 ; 6 ; 18$ & $1 ; 2$ & $1 ; 5$ & $1 ; 4 ; 8$ & 1 & $1 ; 3 ; 4 ; 16 ; 25$ & $1 ; 2 ; 6$ \\
\hline $\begin{array}{c}\text { Ventura y } \\
\text { Noronha } \\
(2014)\end{array}$ & $4 ; 5$ & 1 & $1 ; 5$ & $1 ; 4 ; 8$ & 1 & $4 ; 9 ; 12$ & $1 ; 3$ \\
\hline $\begin{array}{c}\text { Witko, } \\
\text { Bernes, } \\
\text { Magnusson } \\
\text { y Bardick } \\
(2006)\end{array}$ & $2 ; 9$ & $1 ; 2$ & $1 ; 5$ & $1 ; 4 ; 8$ & 1 & $1 ; 2 ; 25$ & $1 ; 4 ; 5$ \\
\hline
\end{tabular}

\section{Discusión y conclusiones}

En este trabajo se ha desarrollado una revisión sistemática de la literatura existente sobre la ODC que en las últimas décadas ha experimentado un paulatino crecimiento de publicaciones en diversos contextos y grupos de población. El propósito de la revisión era identificar las dimensiones y variables fundamentales y pertinentes para el diseño de instrumentos de recogida de información sobre ODC en diferentes edades y/o momentos evolutivos.

Debido a la gran heterogeneidad de estudios, se han acotado los criterios de búsqueda a alumnado de educación secundaria, bachillerato y formación profesional, excluyendo investigaciones centradas en otras etapas educativas, y abarcando las dos primeras décadas del siglo XXI.

Coincidiendo con la revisión empírica desarrollada por Blanco (2009) los estudios analizados reflejan la variabilidad de objetivos de estudio presentes en las investigaciones sobre ODC. No obstante, la mayoría coinciden en escoger como objeto central de estudio el proceso de toma de decisiones vocacionales. Incluso aquellos estudios que no tienen este tópico como objetivo principal, coinciden en señalar la indisoluble relación existente entre la construcción del proyecto de vital de las personas, especialmente en la adolescencia, abarcando las vertientes cognitiva, emocional y social (Álvarez Justel, 2019) y la reflexión para tomar decisiones acertadas y acordes a las preferencias, cualidades, aspiraciones y competencias personales de cada individuo (Montanero, 2000; Rodríguez-Menéndez, Torío-López y Fernández-García, 2006; Álvarez González, 2010; Álvarez González y Bisquerra, 2012). La toma de decisiones constituye uno de los procesos centrales de la ODC, hecho que se pone de manifiesto en las investigaciones revisadas y, por tanto, ha de incluirse de manera ineludible en los elementos a incorporar a los instrumentos de recogida de información tanto cuantitativa como cualitativa, incorporando la perspectiva de género. 
Otro de aspecto destacable es la prevalencia de estudios que enfatizan los factores que influyen en las decisiones de los jóvenes durante la construcción de su proyecto vital, destacando sobre todo las influencias sociofamiliares (Turner y Lapan, 2002; Hargrove, Inman y Crane, 2005; Torío, Hernández y Peña, 2007; Olaosebikan y Olusakin., 2014). Teniendo en consideración las diferentes variables intervinientes en las elecciones académico-profesionales, los adolescentes precisan contar con los apoyos necesarios para enfrentarse a dudas y conflictos y realizar elecciones conscientes y responsables según sus posibilidades e intereses específicos (Moura y Silveira., 2002). Tal y como puede comprobarse en esta revisión, el entorno familiar (padres, hermanos, tíos, abuelos...) y social (amigos, compañeros...) que rodea a los adolescentes son señalados en diversas investigaciones como dos de los factores de mayor impacto e influencia durante los procesos de elección y toma de decisiones sobre sus estudios y su futuro. Es, por lo tanto, un aspecto a incluir en los instrumentos de recogida de información.

La revisión ha mostrado que la mayoría de las investigaciones se han llevado a cabo con estudiantes de educación secundaria obligatoria y bachillerato, pero escasean los estudios sobre ODC con muestras configuradas por estudiantes de formación profesional, modalidad formativa que está llamada a cobrar protagonismo en los próximos años por su capacidad para dar una respuesta de calidad al sistema productivo (García y Romero, 2011). Asimismo, predominan las investigaciones en las que los informantes son únicamente estudiantes $\mathrm{y}$, sin embargo, algunos estudios han aportado evidencias sobre la importancia de la influencia de la familia en las decisiones vocacionales de los adolescentes (Fernández-García, García-Pérez, y Rodríguez-Pérez, 2016) y la trascendencia que tienen los orientadores y las orientadores para un correcto desarrollo del proceso en las distintas etapas educativas, especialmente en secundaria (MartínezClares, Pérez-Cusó, y Martínez-Juárez, 2014). Por eso, se estima pertinente incorporar las voces de otros agentes involucrados en estos procesos.

En cuanto a los diseños de investigación, se caracterizan, en general, por su homogeneidad, predominando los estudios no experimentales. En los últimos tiempos, se está produciendo un incremento de investigaciones basadas en diseños mixtos (Creswell y Plano, 2017). Estos diseños mixtos demandan la utilización de diferentes estrategias que permitan, tanto en el fondo como en el procedimiento, disponer de información recabada desde fuentes diferentes que enriquezcan y complementen la información aportada y los investigadores dispongan de más y mejores datos. En este sentido, deben plantearse diseños de investigación que incorporen diferentes estrategias, instrumentos y técnicas de recogida de información y análisis de datos.

Asimismo, debe destacarse, en relación con los instrumentos de recogida de información en ODC, el predominio de los cuestionarios, en los que las dimensiones tratadas abarcan diferentes elementos cognitivos, emocionales y sociales, cuyos datos se analizan desde la perspectiva cuantitativa y cualitativa. En aras a ampliar y completar el conocimiento de los procesos de diseño, autoconocimiento, etc..., y ayudar al alumnado a diseñar proyectos de vida dinámicos (Romero, 2004) parece recomendable extender el abanico de técnicas e instrumentos de recogida de información y sumar a los cuestionarios otros recursos como los grupos focales, entrevistas, autoinformes, etc., que permitan otorgar la voz al alumnado, familias y profesionales involucrados en la acción orientadora. 
Consecuentemente con el tipo de instrumento utilizado y con los datos recogidos, la mayoría de los análisis son de tipo cuantitativo, y sólo dos investigaciones recurren al análisis de contenido como técnica de análisis (Rodríguez Menéndez, Torío López y Fernández García, 2016). Por el contrario, en el resto de las investigaciones, o bien se combina el empleo de análisis estadísticos y de contenido (Gibbons et al., 2006; Witko et al., 2006; Safta., 2015; Rochat y Rossier, 2016), o emplean únicamente el análisis estadístico para analizar los datos obtenidos.

Consideramos conveniente apuntar algunas de las limitaciones del presente estudio de revisión. Creemos oportuno destacar que podría reconsiderarse algún criterio de inclusión y/o de exclusión en las búsquedas en aras a reducir el riesgo de exclusión de trabajos significativos, por tratarse de documentos ubicados en fuentes secundarias, o por el hecho de que los autores de los estudios primarios hayan empleado en el título, resumen o palabras clave otros términos diferentes, aunque asimilables a la familia semántica. Además, los mismos criterios han podido dejar relegados a exclusión trabajos que, aun conteniendo información fundamental, por estar publicados en un periodo temporal anterior al fijado como criterio de inclusión, hayan sido eliminados de la revisión. Asimismo, han podido dejar de ser considerados otros trabajos por estar escritos en idiomas no contemplados, o por tratarse de documentos como tesis doctorales o publicaciones derivadas de congresos o reuniones científicas, así como trabajos realizados con muestras de etapas educativas previas o posteriores a la educación secundaria pre y post obligatoria o a la formación profesional. Estas limitaciones podrán ser superadas en futuros estudios ampliando el periodo analizado ya que se han podido quedar excluidas investigaciones interesantes, y abriendo el campo del tipo o soporte de los documentos.

Como propuesta para futuros estudios, el análisis de trabajos científicos realizados sobre ODC en las primeras décadas del presente siglo pone de relieve la necesidad de abordar las investigaciones futuras desde una metodología mixta que integre información cuantitativa y cualitativa, utilizando para ello, estrategias e instrumentos de recogida de información que den voz a los participantes. También sería conveniente ampliar las muestras a estudiantes de formación profesional y tener en cuenta a los grupos más vulnerables, como personas con diversidad funcional, a diferentes grupos étnicos o culturales, considerando siempre la perspectiva de género. El contexto sociosanitario provocado por la pandemia de la COVID ha puesto en evidencia las múltiples dificultades y problemas de algunos sectores de la población, que en el futuro van a requerir de más apoyos educativos y de intervenciones orientadoras en todos los ámbitos para que no queden excluidos del sistema productivo y, por tanto, de la vida social (CEDEFOP, 2020). Los profesionales de la ODC han de tener presente tanto en su práctica profesional como en sus investigaciones estas premisas.

\section{Referencias}

Abubakar, I.A. (2018). Career Guidance, Participation of Students and its Implication for Kano, Nigeria. MOJES-Malaysian Online Journal of Educational Sciences, 1(3), 14-19. Álvarez González, M. (2010). La madurez para la carrera. Una prioridad en la educación secundaria. Revista Electrónica de Investigación Psicoeducativa, 6(1), 339-356. https:// doi: 10.25115/ejrep.v6i16.1301 
Álvarez González, M., Figuera Gazo, P. y Torrado Fonseca, M. (2011). La problemática de la transición Bachillerato-Universidad en la Universidad de Barcelona. Revista Española de Orientación y Psicopedagogía, 22(1), 15-27. https://doi:10.5944/reop.vol.22. num.1.2011.74

Álvarez González, M. y Bisquerra Alzina, R. (2017). Orientación educativa. Modelos, áreas, estrategias y recursos. Wolters Kluwer Educación.

Álvarez Justel, J. (2019). Las dimensiones cognitiva, emocional y social en la toma de decisiones de la carrera en el alumnado de educación secundaria. Revista Española de Orientación y Psicopedagogía, 30(3), 140-153. https://doi.org/10.5944/reop.vol.30. num.3.2019.26277

Ambiel, R.A.M., Moreira, T.D.C., Oliveira, D.A., Pereira, E.C. y Hernández, D.N. (2018). Self-efficacy, adaptability and intention of searching for vocational guidance in adolescents. a8. https://doi:10.1590/1982-4327e2840

Bacanli, F. (2016). Career decision-making difficulties of Turkish adolescents. International Journal for Educational and Vocational Guidance, 16(2), 233-250. https://doi:10.1007/ s10775-015-9304-8

Blanco, A. (2009). El modelo cognitivo social del desarrollo de la carrera: revisión de más de una década de investigación empírica. Revista de Educación, 350, 423-445.

Bloxom, J.M., Bernes, K.B., Magnusson, K.C., Gunn, T.T., Bardick, A.D., Orr, D.T. y McKnight, K.M. (2008). Grade 12 student career needs and perceptions of the effectiveness of career development services within high schools. Canadian Journal of Counselling, 42(2), 79-100.

Bounds, P.S. (2017). Contextual factors related to African American adolescent career development. The Career Development Quarterly, 65(2), 131-144. https://doi:10.1002/ cdq.12087

Cedefop; European Commission; ETF; ICCDPP; ILO; OECD; UNESCO (2020). Career guidance policy and practice in the pandemic: results of a joint international survey - June to August 2020. Publications Office of the European Union. http://data.europa.eu/ doi/10.2801/318103

Cenkseven-Önder, F., Kırdök, O. y Işık, E. (2010). High school students' career decisionmaking pattern across parenting styles and parental attachment levels. Electronic Journal of Research in Educational Psychology, 8, 263-280. https://doi:10.25115/ejrep. v8i20.1379

Creswell, J.W y Plano, V.L. (2017). Designing and Conducting Mixed Methods Research. Sage.

Dogar, A.H., Azeem, M., Majoka, M.I., Mehmood, A. y Latif, S. (2011). Need assessment of students' guidance and counseling. British Journal of Arts and Social Sciences, 1(2), 108-124.

Draaisma, A., Meijers, F. y Kuijpers, M. (2018). The development of strong career learning environments: the project 'Career Orientation and Guidance' in Dutch vocational education, Journal of Vocational Education \& Training, 70 (1), 27-46, https:// doi:10.1080/13636820.2017.1392995

Dudovitz, R.N., Chung, P.J., Nelson, B.B. y Wong, M.D. (2017). What do you want to be when you grow up? Career aspirations as a marker for adolescent well-being. Academic Pediatrics, 17(2), 153-160. https://doi:10.1016/j.acap.2016.08.006 
Faria, L.C. (2013). Influencia da condicao de emprego/desemprego dos pais na exploracao e indecisao vocacional dos adolescentes. Psicologia: Reflexao e Critica, 26(4), 772-780. https://doi:10.1590/S0102-79722013000400018

Ferrari, L., Ginevra, M.C., Santilli, S., Nota, L., Sgaramella, T.M. y Soresi, S. (2015). Career exploration and occupational knowledge in Italian children. International Journal for Educational and Vocational Guidance, 15(2), 113-130. https://doi:10.1007/ s10775-015-9299-1

Figuera, P., Rodríguez, M.L y Llanes, J. (2015). Transición y orientación: interrelaciones, estrategias y recomendaciones desde la investigación. REIRE: Revista d'Innovació $i$ Recerca en Educació, 8(2), 1-17.

Fink, A. (2019). Conducting Research Literature Review. Sage.

Frías-Navarro, D. y Monterde-i-Bort, H. (2018). Revisiones sistemáticas: introducción al meta-análisis. En D. Frías-Navarro, M. Pascual-Soler, L. Badenes-Ribera y H. Monterde-i-Bort. Reforma estadística en Psicología. Palmero Ediciones.

García, S. y Romero, S. (2011). La orientación en la formación profesional: una necesidad urgente. Revista Educaçao Skepsis, 2, 1066-1084.

Gibbons, M., Borders, L., Wiles, M., Stephan, J. y Davis, P. (2006). Career and college planning needs of ninth graders - as reported by ninth graders. Professional School Counseling, 10(2), 168-178.

Hernández-Franco, V. (2004). Expectativas vocacionales de resultados en los estudiantes de secundaria. Revista de Investigación Educativa, 22 (1), 89-112.

Hargrove, B.K., Inman, A.G. y Crane, R.L. (2005). Family interaction patterns, career planning attitudes, and vocational identity of high school adolescents. Journal of Career Development, 31(4), 263-278. https://doi:10.1177/089484530503100404

Hirschi, A., Niles, S.G. y Akos, P. (2011). Engagement in adolescent career preparation: social support, personality and the development of choice decidedness and congruence. Journal of Adolescence, 34(1), 173-182. https://doi:10.1016/j.adolescence.2009.12.009

Inda, M., Rodríguez, M.C. y Torío, S. (2017). Evaluación del modelo cognitivo social de desarrollo de la carrera para la predicción de las metas en las materias tecnológicas de estudiantes de bachillerato. Estudios sobre Educación, 32, 49-71. https:// doi:10.15581/004.32.49-71

Karacan-Ozdemir, N. (2019). Associations between career adaptability and career decision-making difficulties among Turkish high school students. International Journal for Educational and Vocational Guidance, 1-21. https://doi:10.1007/s10775-019-09389-0

Königstedt, M. y Taveira, M.C. (2010). Exploração vocacional em adolescentes: avaliação de uma intervenção em classe. Paidéia, 20(47), 303-312. https://doi:10.1590/ S0103-863X2010000300003

Lomelí-Parga, A., López-Padilla, M. y Valenzuela-González, J. (2016). Autoestima, motivación e inteligencia emocional: tres factores influyentes en el diseño exitoso de un proyecto de vida de jóvenes estudiantes de educación media. Revista Electrónica Educare, 20(2), 1-22. https://doi:http://dx.doi.org/10.15359/ree.20-2.4

Lozano, S. y Repetto, E. (2007). Las dificultades en el proceso de decisión vocacional en relación con: el género, el curso académico y los intereses profesionales. Revista Española de Orientación y Psicopedagogía, 18(1), 5-16. 
Marcionetti, J. (2014). Factores que afectan la indecisión de la carrera de los adolescentes en el sur de Suiza. Procedia-Ciencias Sociales y del Comportamiento, 112, 158-166. https://doi:10.19083/tesis/624526

Maree, J.G., Cook, A.V. y Fletcher, L. (2018). Assessment of the value of group-based counselling for career construction. International Journal of Adolescence and Youth, 23(1), 118-132. https://doi:10.1080/02673843.2017.1309324

Martínez-Clares, P. y Martínez-Juárez, M. (2011). La orientación en el s. XXI. Revista Electrónica Interuniversitaria de Formación del Profesorado, 14 (1), 253-263.

Martínez-Clares, P., Pérez-Cusó, F.J. y Martínez-Juárez, M. (2014). Orientación profesional en educación secundaria. Revista Electrónica Interuniversitaria de Formación del Profesorado, 17(1). https://doi:10.6018/reifop.17.1.198841

Morán, G., Muñoz, T. y García, Y.K. (2015). Las perspectivas de los estudiantes de bachillerato para su orientación vocacional. Revista de Docencia e Investigación Educativa, 1(2), 101-110.

Moura, C.B. y Silveira, J.M. (2002). Orientação profissional sob o enfoque da análise do comportamento: avaliação de uma experiência. Estudos de Psicologia, 19(1), 5-14.

Núñez, E. (2010). Los incentivos que motivan a los jóvenes a estudiar formación profesional: cómo mejorar su horizonte educativo con el marketing. Prisma Social: Revista de Investigación Social, 4, 1-34.

Olaosebikan, O.I. y Olusakin, A.M. (2014). Effects of parental influence on adolescents' career choice in Badagry Local Government Area of Lagos State, Nigeria. Journal of Research \& Method in Education, (4), 44-57. https://doi:10.9790/7388-04434457

Olivares, M.A., de León, C. y Gutiérrez, P. (2010). El proceso de orientación profesional en los institutos de educación secundaria. El caso de Córdoba. Revista Española de Orientación y Psicopedagogía, 21(1), 81-92. https://doi:10.5944/reop.vol.21. num.1.2010.11514

Paixão, O. y Gamboa, V. (2017). Motivational profiles and career decision making of high school students. The Career Development Quarterly, 65(3), 207-221. https:// doi:10.1002/cdq.12093

Planas, J.A. (2012). La orientación profesional y la búsqueda de empleo. Graó.

Rochat, S. y Rossier, J. (2016). Integrating motivational interviewing in career counseling: a case study. Journal of Vocational Behavior, 93, 150-162. https://doi:10.1016/j. jvb.2016.02.003

Rodríguez-Menéndez, M.C., Peña-Calvo, J.V. y Inda-Caro, M. (2016). “Esto es lo que me gusta y lo que voy a estudiar": un estudio cualitativo sobre la toma de decisiones académicas en bachillerato. Revista Complutense de Educación, 27(3), 13-51. https:// doi:10.5209/revrced.2016.v27.n3.48518

Rodríguez-Menéndez. C., Torío-López, S. y Fernández-García, C.M. (2006). El impacto del género en las elecciones académicas de los estudiantes asturianos que finalizan la ESO. Revista Española de Orientación y Psicopedagogía, 17(2), 239-260.

Rodríguez Moreno, M.L. (2013). Orientar y orientarse en época de crisis e incertidumbre: narrar la experiencia profesional. En P. Figuera Gazo (coord.), Orientación profesional $y$ transiciones en el mundo laboral, p.111-160. Laertes.

Romero, S. (2004). Aprender a construir proyectos profesionales y vitales. Revista Espanola de Orientación y Psicopedagogía, 15(2), 337-354. 
Safta, C.G. (2015). Career decisions. A test of courage, responsibility and self-confidence in teenagers. Procedia-Social and Behavioral Sciences, 203, 341-347. https://doi:10.1016/j. sbspro.2015.08.305

Salami, S.O. y Oyesoji-Aremu, A. (2007). Impact of parent-child relationship on the career development process of high school students in Ibadan, Nigeria. Career Development International, 12(7), 596-616. https://doi:10.1108/13620430710834387

Santana, L.E. y Feliciano, L.A. (2011). Percepción de apoyo de padres y profesores, autoconcepto y toma de decisiones en bachillerato. Revista de Educación, 355, 493519. https://doi:10-4438/1988-592X-RE-2011-355-034

Santana, L.E., Feliciano, L.A. y Santana-Lorenzo, J.A. (2012). Análisis del proyecto de vida del alumnado de educación secundaria. Revista Española de Orientación y Psicopedagogía, 23(1), 26-38. https://doi:10.5944/reop.vol.23.num.1.2012.11391

Santana, L.E., Alonso, E. y Feliciano, L. (2018). Trayectorias laborales y competencias de empleabilidad de jóvenes nacionales e inmigrantes en riesgo de exclusión social. Revista Complutense de Educación, 29(2), 355-369. https://doi:http://dx.doi. org/10.5209/ RCED.52444

Savickas, M. (2012). Life design: a paradigm for career intervention in the 21st century. Journal of Counseling and Development, 90, 13-19.

Tang, M., Pan, W. y Newmeyer, M. (2008). Factors influencing high school students' career aspirations. Professional School Counseling, 11(5), 285-295.

Taveira, M.C. y Rodríguez-Moreno, M.L. (2010). La gestión personal de la carrera y el papel de la orientación profesional: teoría, práctica y aportaciones empíricas. Revista Española de Orientación y Psicopedagogía, 21(2), 335- 345. https://doi:10.5944/ reop.vol.21.num.2.2010.11536

Torío, S., Hernández, J. y Peña, J.V. (2007). Capital social familiar y expectativas académico-formativas y laborales en el alumnado de educación secundaria obligatoria. Revista de Educación, 343, 559-586.

Turner, S. y Lapan, R.T. (2002). Autoeficacia profesional y percepciones del apoyo de los padres en el desarrollo de la carrera de los adolescentes. The Career Development Quarterly, 51(1), 44-55.

Vázquez, I.M. y Blanco, A. (2019). Factores sociocognitivos asociados a la elección de estudios científico-matemáticos. Un análisis diferencial por sexo y curso en la educación secundaria. Revista de Investigación Educativa, 37(1), 269-286. https:// doi:http://doi.org/dwfg

Ventura, C.D. y Noronha, A.P.P. (2014). Autoeficácia para escolha profissional, suporte familiar e estilos parentais em adolescentes. Avaliaçao Psicologica, 13(3), 317-324.

Witko, K.D., Bernes, K.B., Magnusson, K.C. y Bardick, A.D. (2006). Senior high students' career plans for the future: outcomes of the comprehensive career needs survey in Southern Alberta, Canada. International Journal for Educational and Vocational Guidance, 6(2), 77-94. https://doi:10.1007/s10775-006-9103-3

Fecha de recepción: 9 de junio de 2020.

Fecha de revisión: 2 de julio de 2020.

Fecha de aceptación: 17 de marzo de 2021. 